\title{
Bridges to SCANDINAVIA
}

Edited by Andrea Meregalli and Camilla Storskog

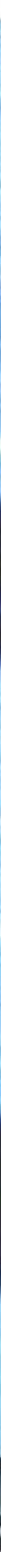






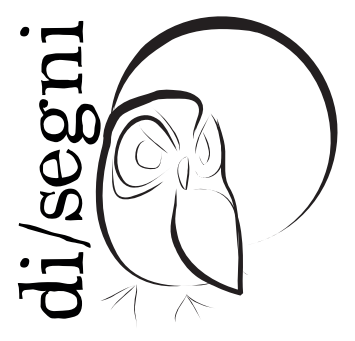





\section{Bridges to ScandinaVia}

Edited by Andrea Meregalli and Camilla Storskog

di/segni

Dipartimento di Lingue e Letterature Straniere

Facoltà di Studi Umanistici

Università degli Studi di Milano 
(C) 2016 degli autori dei contributi e di Andrea Meregalli e Camilla Storskog per l'insieme del volume

ISBN 978-88-6705-4I2-I

ILLUSTRAZIONE DI COPERTINA:

The Øresund Bridge

Elaborazione grafica su fotografia di Flora Cusi

$\mathrm{n}^{0} 15$

Collana sottoposta a double blind peer review

ISSN: 2282-2097

\title{
Grafica:
}

Raúl Díaz Rosales

\section{Composizione:}

Ledizioni

Disegno del logo:

Paola Turino

\author{
STAMPATO A MILANO \\ NEL MESE DI GIUGNO 2016 \\ www.ledizioni.it \\ www.ledipublishing.com \\ info@ledizioni.it \\ Via Alamanni 11 - 20141 Milano
}

\begin{abstract}
Tutti i diritti d'autore e connessi sulla presente opera appartengono all'autore. L'opera per volontà dell'autore e dell'editore è rilasciata nei termini della licenza Creative Commons 3.0, il cui testo integrale è disponibile alla pagina web http://creativecommons.org/licenses/by-sa/3.0/it/legalcode
\end{abstract}




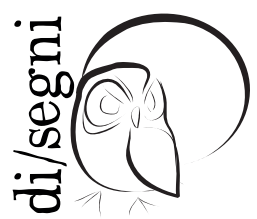

Direttore

Emilia Perassi

\title{
Comitato scientifico
}

\author{
Monica Barsi Francesca Orestano \\ Marco Castellari Carlo Pagetti \\ Danilo Manera Nicoletta Vallorani \\ Andrea Meregalli Raffaella Vassena
}

\section{Comitato scientifico internazionale}

Albert Meier Sabine Lardon

(Christian-Albrechts-Universität zu Kiel) (Université Jean Moulin Lyon 3)

Luis Beltrán Almería Aleksandr Ospovat- Александр Осповат (Universidad de Zaragoza) (Высшая Школа Экономики - Москва)

Patrick J. Parrinder

(Emeritus, University of Reading, UK)

\section{Comitato di redazione}

Laura Scarabelli (coordinatrice)

Nicoletta Brazzelli Simone Cattaneo

Margherita Quaglia Sara Sullam 


\title{
L'EUROPA DEI TEATRI E I BARBARI SCANDINAVI
}

\author{
Franco Perrelli \\ UnIVERSITÀ DEGLI STUdI DI ToRINO
}

I. Non è la prima volta che ci si propone di mettere a fuoco l'affermazione sul continente dei grandi drammaturghi nordici alla fine dell'Ottocento, ma - anche se spesso si sono evidenziate le reazioni (e pure gli ostacoli) sul loro cammino - l'esito conclusivo della loro penetrazione nella cultura europea ha un po' attenuato il rilievo della contingente effettiva portata del loro successo, che, all'analisi, risulta quantomeno un processo faticoso, discontinuo e tutt'altro che condiviso a livello di critica e di pubblico.

Sarà opportuno quindi rivisitare alcuni passaggi di questa vicenda per disegnare con maggiore oggettività i problemi e i limiti di comprensione che, in Germania come in Francia, l'apparizione sulle scene di Bjørnson, Ibsen e soprattutto Strindberg comportò e per cogliere alcune interessanti scissioni identitarie e culturali dell'Europa fra il XIX e il XX secolo.

2. "Germania o Francia? Non ci sarebbe da scrivere un libro?", così s'interrogava il filofrancese Strindberg, qualche anno prima di scegliere di stabilirsi in Germania (ASB VIII, I47). ${ }^{.}$Certo è che le aree culturali francese e tedesca, i tanti teatri delle loro capitali (e, in Germania, pure di centri importanti come Monaco, Dresda, Lipsia, Meiningen ecc.), i loro traduttori e le agenzie attive in quelle nazioni erano i canali obbligati per l'affermazione degli autori nordici sul continente.

La tendenza si era vieppiù configurata quando la nota compagnia del Duca di Meiningen (appassionato della drammaturgia scandinava fino a

I Le lettere e le opere di August Strindberg saranno d'ora in poi citate, nel testo, con i seguenti acronimi: ASB per Strindberg 1947-200I; ASV per Strindberg I98I-2013. Le traduzioni sono di chi scrive, ove non altrimenti specificato. 
spingersi alla ripresa di Ludvig Holberg) aveva proposto nella prima tournée berlinese del I874 Fra le battaglie (Mellem slagene) di Bjørnstjerne Bjørnson. ${ }^{2}$ Pur presentato, con un evidente effetto di contrasto, in singolare accoppiata con Le Malade imaginaire di Molière, l'allestimento, secondo il programma dei Meininger, era stato un esempio del più attento realismo, e il Duca aveva chiesto allo scenografo Max Brückner "un basso fortilizio con porte strette e un pavimento coperto di canne", curando che in scena ardessero autentici ceppi e che si udisse l'ululare del vento (Koller i984, I56).

Insomma, ricorda l'attore Max Grube, nel suo volumetto di memorie sui Meininger del ig26: "Prima della leggerezza e della grazia molièriane, la pesantezza, il ritmo grave e lento!”, e, se Zwischen den Schlachten, con la sua evocazione del "tenebroso brumoso mondo nordico", non fu un successo ("interessò e tuttavia parve troppo strano"; Grube i963, 68), restò comunque in repertorio. L'autorevole critico Karl Frenzel gli avrebbe quantomeno riconosciuto "il fascino e la fresca consistente originalità dei racconti" di Bjørnson, già noti in Germania (Osborne I988, 72; Eller I9ı8, 23).

L'attenzione verso gli autori scandinavi si rafforzò allorché anche Ibsen (che soggiornò a lungo a Dresda e a Monaco) cominciò a farsi conoscere, in prima battuta con le opere giovanili, che - sempre per il loro romantico colore nordico-arcaico - potevano essere in sintonia con un certo gusto pittorico-antiquario diffuso nell'arte tedesca dell'epoca. ${ }^{3}$

Così, allo Hoftheater di Monaco, il ıo aprile ı876, fu allestita quella che si considera la prima rappresentazione ibseniana fuori della Scandinavia: I condottieri a Helgeland (Hormoendene på Helgeland, I858). Il giorno dopo, Ibsen comunicava all'editore Hegel:

La sala era praticamente piena e il dramma è stato accolto da una tempesta d'applausi. Io ho seguito la rappresentazione dietro le quinte e poi sono stato acclamato cinque volte. Dopo lo spettacolo, i letterati monacensi hanno improvvisato una festa in mio onore che si è protratta nella notte (HIS XIII, 308). ${ }^{4}$

Sin dalla breve ma significativa premessa all'edizione tedesca del testo (Nordische Heerfahrt, tradotto da Emma Klingenfeld) del marzo ı876, Ibsen ringraziava il suo "stimatissimo amico" istruttore dello Hoftheater, Franz Grandaur, e sottolineava (certo strumentalmente) l'affinità 'pangermanica' della propria opera:

2 "Nel I867, [i Meininger] avevano presentato la prima produzione tedesca di Bjørnson, Fra le battaglie, e, due anni dopo, il debutto assoluto e integrale della trilogia di Sigurd Slembe" (Koller i984, 87).

3 Il primo dramma di Ibsen tradotto e pubblicato in volume in Germania era stato Brand, nel febbraio del 1872 .

4 Citeremo, nel testo, le lettere ibseniane con l'acronimo HIS, traendole da Ibsen 2005-Io. 
Il fondamento sostanziale del mio dramma sta di fatto soprattutto nelle diverse saghe familiari islandesi che ci sono pervenute, nelle quali molto spesso le celebri colossali situazioni e gesta del Nibelungenlied e della Saga dei Volsunghi paiono ricondotte a dimensioni umane. Credo pertanto di poter concludere che le vicende e gli avvenimenti narrati in ambedue le opere citate siano stati caratteristici di tutta la nostra vita germanica nelle epoche protostoriche (Ibsen I909, I, 300-OI).

Il 30 maggio di quell'anno fortunato, Ibsen poteva ancora annunciare al suo editore:

Ho ricevuto in questo momento l'invito dal Duca di Meiningen di recarmi a Berlino, dove sabato la sua compagnia darà per la prima volta I pretendenti alla corona. [...] È falso quello che un giornale danese ha riportato ovvero che il dramma sarebbe stato rappresentato a Meiningen senza un applauso. Si trattava solo di una prova generale [tenuta il 30 gennaio 1876 ] priva della nuova scenografia e dei miei tagli (HIS XIII, 3I2-I3).

I pretendenti alla corona (Kongs-emnerne), risalenti al I863, avevano attirato l'attenzione dei Meininger, parrebbe, per suggerimento di Bjørnson (autore sempre molto vicino alla compagnia), oltre che per la loro pittoresca ambientazione medievale (Koller I965, IOI).

Il 3 giugno I876, Die Kronprätendenten fu presentato a Berlino e, il I4, Ibsen poteva raccontare all'istruttore svedese Ludvig Josephson, che da poco aveva allestito a Christiania un memorabile Peer Gynt: "Al principio del mese, mi sono recato a Berlino per assistere alla prima dei Pretendenti alla corona montati con gusto splendido dalla compagnia di corte del Duca di Meiningen”. Quindi:

Il dramma è stato accolto con grandi applausi e io sono stato acclamato parecchie volte. Essendo la maggior parte dei critici berlinesi dei drammaturghi, non credo che la cosa facesse loro troppo piacere. Il dramma, in ogni caso, è stato dato per nove sere di fila e sarebbe andato anche più a lungo se i Meininger non avessero dovuto concludere le rappresentazioni già il I5. Dopo la prima, sono stato invitato dal Duca nel suo castello di Liebenstein, nei pressi di Meiningen, dove mi sono trattenuto sino all'altro ieri per poi far ritorno qui [a Monaco]. Al momento del congedo, sono stato decorato con la Croce di Prima Classe del casato sassone-ernestino. I pretendenti saranno ora messi in scena a Schwerin e I condottieri a Helgeland sono programmati 
al Burgtheater di Vienna, dove Charlotte Wolter reciterà Hjørdis (HIS XIII, 3I3-I4).

Max Grube fu meno entusiasta di Ibsen e ricorda che "I pretendenti non ebbero più successo di Fra le battaglie di Bjørnson”, per il loro verboso stile epico e per l'equivalente peso morale dei due protagonisti in lotta per la corona, che impedisce al pubblico di schierarsi e simpatizzare con uno di loro. Ciò detto,

il Duca in persona aveva prodigato molta attenzione alla messinscena dei Pretendenti. Nel corso di un viaggio nella patria di Ibsen - all'epoca poco visitata - aveva studiato attentamente il carattere del paese. Alcuni dei suoi disegni sono rimasti. Lì aveva inoltre rinvenuto abbondanza di fonti per quanto riguardava $i$ costumi. Questa peculiarità esotica esibita dalla rappresentazione sollecitò un così ampio interesse che, nonostante la debolezza del dramma, lo si diede sette volte (Grube ig63, 8I).

(Ovvero due di meno di quelle indicate da Ibsen.) Dai bozzetti dello stesso Duca apprendiamo che le scene erano massicce e grigie e i costumi realizzati con pesanti tessuti dalle cupe sfumature blu e rosse (Koller I965, IOI). La Scandinavia, a livello figurativo e d'atmosfera, si fissava come un cosmo arcaico, immancabilmente grave e brumoso, affascinante per la sua intrinseca cupezza.

Nel complesso, anche la critica su I pretendenti si tenne riservata. Su Deutsche Rundschau, Karl Frenzel ritenne Ibsen un autore in grado di esprimere movimento e personalità drammatiche, carente tuttavia sul piano della costruzione dell'opera. Il giudizio del critico appare sostanzialmente negativo: "Si perde tempo, denaro ed energie a sforzarsi di presentare drammi che, nonostante il loro valore poetico, non potranno mai naturalizzarsi sulle scene tedesche a causa delle loro caratteristiche di fondo che ci sono assolutamente estranee" (Eller I9I8, 32).5 Il tentativo di Ibsen d'integrarsi come tedesco incontrava così i primi ostacoli.

3. Sebbene l'esame della stampa berlinese faccia rilevare, in quell'epoca di nazionalismi ben rimarcati, delle radicate diffidenze verso la dilagante passione per gli autori nordici, con la relativa evidenziazione di affermazioni in fondo assai tiepide per Ibsen, l'autore norvegese cominciava comunque a farsi strada in Germania, come si evince da una lettera del i5 settembre I876 al suo editore. In essa, infatti, Ibsen può confermare per ottobre-novembre le messinscene annunciate a Josephson e aggiungere:

5 In un articolo su National-Zeitung, Frenzel aveva invece lodato l'allestimento dei Meininger, caratterizzato da "figurazioni brillanti e scene avvincenti" (vedi Koller I965, I02-I3). 
I condottieri a Helgeland saranno allestiti allo Hoftheater di Dresda entro un paio di settimane; lo stesso testo è in prova allo Stadttheater di Lipsia e, attualmente, replicato con successo a Monaco. Da ognuno di questi teatri riceverò a vita il ı०\% dei ricavi delle rappresentazioni e i miei eredi lo stesso per I5 anni (HIS XIII, 323-24).

Si trattava di condizioni vantaggiose, in una nazione nella quale il diritto d'autore era un principio ancora incerto. Per di più si stava profilando la prospettiva di vedere forse allestito, a Meiningen e a Monaco, un altro dramma giovanile, Donna Inger di Østråt (Fru Inger til Østråt, I855), che di fatto sarebbe andato in scena al Nationaltheater di Berlino nel dicembre del I878.

In questo periodo, tuttavia, Ibsen si stava proiettando al di là di quella produzione giovanile, che pure stava riscuotendo attenzione, e guardava al teatro con occhi diversi. Sempre nella sua premessa all'edizione tedesca dei Condottieri, puntualizzava, infatti:

Per la messinscena, le figure idealizzate e a un certo grado impersonali del mito oggi si prestano meno che mai; comunque prescindendo in assoluto da ciò, io avevo solo intenzione in generale di presentare [nei Condottieri] la nostra vita nei tempi antichi, non la nostra mitologia (Ibsen I909, I, 30I).

La vita e la storia contro le mitologie appare qui l'enunciazione di un intento realistico; comunque, non sarebbe stato lui, bensì, ancora una volta, il pioniere Bjørnson a rivelare ai tedeschi il primo serio esperimento di 'tragicommedia del denaro' con Bancarotta (En fallit) del I874, opera che, dopo varie vicissitudini in Norvegia, era approdata prima al Nya Teatern di Stoccolma e, quindi, in Germania, nel I875, "coronata da un indubbio successo" e allestita in quasi tutti i teatri della nazione: "La mano che aveva brandito la spada di Sigurd" - sentenzierà Georg Brandes - "non si sentì umiliata a contare il denaro del mercante Tjælde e a ricapitolare le voci dei suoi debiti" (Brandes I977, I58). Quando Ein Fallissement giunse a Berlino (assieme a un antico dramma di Bjørnson come Gli sposini, De nygifte, del I865) fu giudicato da Frenzel “un bozzetto 'grigio su grigio'”, ma da Paul Lindau, su Die Gegenwart, un'opera che suscitava una forte impressione (Eller I9I8, 24).

Nel giugno di quell'anno, anche Ibsen assistette a una rappresentazione di Ein Fallissement a Monaco e la circostanza probabilmente lo stimolò vieppiù a portare a termine Le colonne della società (Samfundets støtter), le quali, a loro volta, dai primi mesi del I878, vennero rappresentate su ben cinque palcoscenici berlinesi, riscuotendo - come ebbe modo di rilevare la stampa - "un successo senza precedenti nelle cronache teatrali della città", ${ }^{6}$ nonché a casca-

6 Così su Nationalzeitung, cfr. l'introduzione bibliografica di Jens Braage Halvorsen (Ibsen I898-I902, VI). 
ta in decine di scene austro-tedesche, quantunque - va sottolineato - largamente in traduzioni scarse: "Tornavamo di continuo nel teatro in cui si dava il dramma e, quando era tutto esaurito, lo leggevamo nella tremenda versione di Wilhelm Lange". Così rievoca Paul Schlenther, uno dei giovani intellettuali che volevano rinnovare il teatro tedesco e che era stato folgorato da Stützen der Gesellschaft: "I nostri occhi si aprirono sul falso ciarpame teatrale che ci veniva offerto. Eravamo entusiasti. [...] Ibsen fino ad allora era stato per noi un nome vuoto. Fu quest'opera che c'insegnò ad amarlo e fu un amore per la vita" (cit. in Meyer I97I, 44I). Critici meno schierati, però, come l'autorevole Frenzel, riconobbero nel testo di Ibsen "ovunque la zampata leonina del poeta, da nessuna parte quella del drammaturgo", ché Le colonne apparivano "un racconto ridotto a forza in forma drammatica" (Eller i9ı8, 33).

Un capitolo a sé dovrebbe poi costituire la storia della pirateria applicata diffusamente a questo testo in area germanica. Pirata era, del resto, "la tremenda versione di Wilhelm Lange", letta da Schlenther, ma ci limiteremo a ricordare che il successo di Ibsen fu nulla rispetto a quello realizzato dallo "spaventoso bandito della letteratura" Emil Jonas (HIS XIII, 422, e anche 420-2I), il cui rimaneggiamento del copione, conforme al "gusto tedesco" come dichiarò su un giornale lo spavaldo adattatore - era stato adottato da ben trentadue teatri (Meyer I97I, 446-47).

4. Il successo di Bancarotta non fu duraturo e il decennio successivo si presentò piuttosto difficile per Bjørnson: i drammi realistici di Ibsen misero in ombra le sue opere per molti versi avanguardistiche e di assoluto rilievo storico, ma più sentimentali ed effimere. Anche in Germania, Casa di bambola (Et dukkehjem) ovvero Nora oder Ein Puppenheim, rappresentata per la prima volta al Fleensburg Stadttheater e sulle principali scene tedesche dal febbraio del I880, suscitò un acceso dibattito, stimolando contingenti contributi critici (Lindau e Frenzel, pur dimostrando qualche considerazione per l'autore, trovarono il dramma poco credibile, se non sbagliato), ma anche più approfondite prese di posizione come l'intervento di Eugen Zabel su Unsere Zeit (che legava il norvegese a Goethe, al pessimismo, oltre che alla discendenza germanica) e di Ludwig Passarge, il quale, nell'83, pubblicò la prima monografia in tedesco di ben 3 Io pagine dedicata al drammaturgo, che sollevò la questione di "quanto importante fosse Ibsen per la Germania” (Eller I9ı8, 33-35, 43-45).

Un altro capitolo a sé costituirebbe la reazione degli interpreti a Casa di bambola, delle attrici protagoniste in particolare, le quali, proprio sulle scene tedesche, costrinsero Ibsen a contrabbandare un singolare lieto fine per il suo dramma, come fu preteso dalla diva Hedwig Niemann-Raabe, ma volentieri accettato pure da Maurice ad Amburgo e da Laube a Vienna (Eller I9I8, 38-39). ${ }^{7}$

7 Ricorderemo incidentalmente che al Residenz-Theater di Berlino, nelle rappresentazioni dell'8o, si manipolò ulteriormente il finale con l'ingresso dei figlioletti, che venivano abbracciati 
Nonostante tutto questo clamore, Ibsen conobbe un certo rallentamento d'interesse da parte dei teatri a partire dal I880, almeno fino al 2I dicembre I886, allorché il Duca di Meiningen fece allestire i suoi scandalosi Spettri (Gengangere, I88I) nel locale Hoftheater, che non era ovviamente sottoposto a censura. ${ }^{8}$ Max Grube (interprete del Pastore Manders) ricorda che il solo annuncio della messinscena di Spettri aveva messo in subbuglio il piccolo ducato di Meiningen, dove non molti avevano letto il testo, ma "era universale opinione che il dramma fosse estremamente indecente e immorale". Gli autori nordici, in fondo, piacevano più al Duca che al suo pubblico ${ }^{9}$ e, in questa occasione, si sollevò una vera e propria protesta e "veniva dato per scontato che tutte le signore sarebbero rimaste a casa". Così non si vendettero biglietti, ma il Duca corse ai ripari distribuendoli tra i suoi funzionari e ufficiali e facendo presidiare il teatro dai pochi poliziotti in forza a Meiningen (Grube I963, I09-I0). Nonostante gli auspici negativi, "molta gente interessata affluì da ogni dove, specie da Berlino" (HIS XIV, 375-76), anche se, in sala, "con l'eccezione di Frau von Heldburg, erano presenti solo poche coraggiose donne del teatro di corte" (Grube I963, IIo). Lo spettacolo si tenne senza incidenti e, considerato che a capo della claque c'era lo stesso Duca, con inevitabile successo.

A parte Grube, Maria Berg interpretò la Signora Alving, Alexander Barthel Osvald e Karl Weiser, attore specializzato nella resa di "una certa brutalità", Engstrand. ${ }^{\text {Io }}$ Erano tutti artisti di turgida tradizione shakespeariana e schilleriana e ci s'interroga su come affrontassero lo spoglio copione ibseniano. Regine ovvero l'attrice Klothilde Schwarz fu comunque reclutata allo Hoftheater di Monaco. Per parte sua, Ibsen aveva fornito ragguagli realistici e pressoché visivi sull'ambientazione norvegese del dramma (cfr. HIS XIV, 365-66) e, in una lettera del I5 novembre I886, il Duca invitò i suoi scenografi di fiducia, i fratelli Brückner, a realizzare un interno con, "sullo sfondo, la vetrata che dava su una serra, che di preferenza doveva ospitare

da Nora, fra i singhiozzi generali. Il dramma, nonostante questo, fu accolto con qualche imbarazzo, secondo Georg Brandes, soprattutto perché il Residenz-Theater era "corrotto da Sardou". Lo stesso anno, l'esito fu contrastato anche al Residenz-Theater di Monaco, dove il ruolo principale fu tenuto da Marie Ramlo, ma almeno adottando il finale originale (cfr. il vol. di commento a HIS VII, 246-48).

8 La prima assoluta di Spettri fu data in Germania, per impulso di Felix Philippi, in forma privata, come prova in costume e senza vendita di biglietti, ma alla presenza di Ibsen, allo Stadttheater di Augusta, il I4 aprile I886. Anche in questa occasione, qualche giornale ricondusse Ibsen al pessimismo di Schopenhauer e Hartmann, nonché soprattutto allo gnosticismo del Manfred di Byron (Koller I965, I05).

9 Pare che il Duca stesso li considerasse delle 'chicche', genere cui apparteneva, tra l'altro, Maria Stuart i Skotland di Bjørnson che veniva rappresentata piuttosto spesso, "quantunque godesse dello stesso scarso favore di Halte Hulda dello stesso autore" (Grube I963, I09).

ro Fu questo attore che, dopo lo spettacolo, chiese a Ibsen se "il falegname zoppo avesse dato davvero fuoco all'asilo, un punto non chiaro nel dramma, che Ibsen sembrò ponderare seriamente prima di rispondere: 'Potrebbe essere così'. Si aveva l'impressione che parlasse non d'una figura d'invenzione, ma di una persona reale" (Grube I963, 96, IIо). 
fiori veri. Attraverso la finestra posteriore si deve vedere un paesaggio norvegese, per il quale si può usare lo spezzato del [Wilhelm] Tell" (Koller ig65, ıо6), secondo una prassi di riciclaggio del materiale scenico tipica dell'Ottocento ed evidentemente non ignota anche ai pur prestigiosi e meticolosi Meininger.

Il Duca nutriva per Gespenster ambizioni di tournée che dovettero drasticamente ridimensionarsi di fronte ai ripetuti dinieghi della censura in varie e poco liberali città tedesche (la produzione fu comunque, nell'immediato, replicata a Meiningen e a Dresda; cfr. HIS XIV, 425). Infine, nel gennaio dell'87, si autorizzarono - piuttosto singolarmente, per beneficenza - il direttore del Residenztheater di Berlino, Anton Anno, e l'istruttore Franz Wallner (che veniva dai Meininger e avrebbe interpretato Osvald) a rappresentare in matinée il testo con Charlotte Frohn nel ruolo della Signora Alving. Qui pare si registrassero fino a $\mathrm{I} 4.000$ richieste d'ingresso (Koht I954, II, 2I8) e il critico Alfred Kerr avrebbe avuto occasione di parlare di uno "dei più significativi eventi teatrali degli ultimi anni", a marcare soprattutto il successo dell'Ibsen realista, per questo apprezzato da un pubblico pur sempre d'avanguardia (cfr. il vol. di commento a HIS VII, 467).

Non a caso, due anni dopo, il copione fu scelto da Paul Schlenther e Otto Brahm per inaugurare la Freie Bühne, la scena sperimentale che seguiva l'esempio del parigino Théâtre Libre di André Antoine. In gioco, tanto per Brahm quanto per Antoine, c'era l'emancipazione del teatro dal dominante modello commerciale francese, e Ibsen, essendo in fondo - secondo la discutibile convinzione del primo - "di sangue germanico", poteva incidere sul teatro tedesco esattamente come aveva fatto a suo tempo Lessing (Meyer I971, 579).

Il I889 fu, del resto, un anno decisivo per la fortuna tedesca di Ibsen, poiché, a Berlino, in vari teatri, si realizzò una specie di festival delle sue opere, come il drammaturgo stesso relazionava alla moglie, il 5 marzo:

\begin{abstract}
Ieri mattina, s'è tenuta la prova generale [della Donna del mare (Fruen fra havet), al Königliches Schauspielhaus], con mia piena soddisfazione. La sera si organizzò una festa maschile in mio onore, presenti I6-I7 persone: Lindau, Bülow e Kainz, tra gli altri. Domani sera, sarà rappresentata Wildente [L'anitra selvatica (Vildanden)] al Residenztheater e dopodomani Nora al LessingTheater di Blumenthal. Sono ancora indeciso se congedarmi da qui o recarmi a Weimar [per Die Frau vom Meere], come mi stanno persuadendo a fare (HIS XIV, 536-37).
\end{abstract}

Calato il sipario su Nora, Ibsen ricevette un'enorme corona d'alloro, che possiamo considerare un simbolo dell'acme della sua fama in Germania. Dopo il colorito romanticismo dei drammi giovanili, il drammaturgo norvegese 
imponeva adesso, nel clima decadente della fine del secolo, anche al di là di un realismo oggettivo, quella che Hofmannsthal definirà una poesia "delle esistenze che si consumano patologicamente", contribuendo, nei paesi di lingua tedesca, all'affermazione di un teatro dall'ambigua cifra natural-simbolista, ritenuto moralmente e politicamente eversivo: "Una tendenza che non mi piace affatto", avrebbe sintetizzato senza troppe sfumature Guglielmo II. ${ }^{\text {II }}$

Tuttavia, dall'insuccesso di Hedda Gabler al Residenztheater di Monaco nel gennaio I89I (che si replicò il mese dopo a Berlino), si avvia in Germania un certo declino della parabola dell'autore norvegese, che si avventurava peraltro, nei suoi estremi drammi, su una strada di scrittura vieppiù sperimentale. Nel I902-03, Otto Weininger osservava:
Per chi si trova su posizioni più avanzate, quel nome indica qual- cosa di banale, una parola d'ordine contrapposta agli epigoni del classicismo. Si crede di aver compreso ormai anche troppo bene ciò che egli ha detto, e di averlo fatto proprio da un bel pezzo. Per costoro egli ha annunciato un'epoca che di fatto è ormai iniziata, e conquiste che sono già patrimonio comune della scienza: e non c'è nulla che più di questo risulti fatale per un'opera d'arte (1985, 8). ${ }^{\text {I2 }}$

5. Fu in tale contesto che pure Strindberg, a partire dalla seconda metà degli anni Ottanta, cercò di farsi strada in Germania, dove - sulla scorta della sua narrativa, pubblicata da un editore di Budapest in odore di pornografia - era quantomeno considerato "uno scrittore svedese sensazionalista", per di più d'ispirazione socialista (Ollén I982, I09). Alla Freie Bühne, in recite private per i soliti pressanti motivi di censura, furono rappresentati Il padre (Fadren, I887) il I2 ottobre I89o, con gli ottimi attori Emanuel Reicher e Rosa Bertens protagonisti, nonché La signorina Julie (Fröken Julie, I888) due anni dopo, il 3 aprile, ancora con Rosa Bertens nello scandaloso ruolo della protagonista e Rudolf Rittner in quello di Jean.

La rappresentazione, già malaccortamente suddivisa in due atti, fu preceduta da una conferenza di Paul Schlenther, il quale si dimostrò imbarazzato di fronte al dramma strindberghiano, troppo spinto, e non evitò il raffronto tra lo svedese e Ibsen (peraltro frequente nella critica del tempo), nell'ambito del

II Si consideri che del I889-90 sono anche Prima dell'alba (Vor Sonnenaufgang) di Hauptmann e Risveglio di primavera (Frühlings Erwachen) di Wedekind (cfr. Melchinger I962, 3I-34).

I2 In ogni caso, Ibsen - si è giustamente osservato - rimase, al di là della propria personale fortuna, un forte elemento ispiratore per quel movimento di rinnovamento realistico e sperimentale, in ambito letterario e drammaturgico, che corrisponde a Junges Deutschland, servendo addirittura come "prototipo per la localizzazione nazionale delle idee all'ordine del giorno". Infatti, il drammaturgo, rendendo universale il microcosmo norvegese, aveva offerto per primo l'esempio di quella che poteva essere una Heimatskunst (Eller I9I8, I25-26). 
quale, a suo avviso, i personaggi della Signorina Julie presentavano, alla fine, il difetto di stagliarsi meno definiti e meditati di quelli del "maestro" norvegese. ${ }^{13}$ Nonostante queste cautele, grazie agli attori, i quali "dimostrarono", come osserverà Adolf Paul (I930, 36), "che un buon dramma non ha bisogno di conferenzieri, ma di essere recitato per bene [...], l'effetto dell'opera fu forte".

Per Strindberg, però, il vero successo berlinese (una settantina di repliche $)^{\mathrm{I} 4}$ giunse il 22 gennaio I893, allorché il Residenztheater diede, sotto la direzione di Siegmund Lautenburg, Creditori (Fordringsägare, I888). Ricorda ancora Adolf Paul che un esito così felice si verificò del tutto inaspettato:

In teatro non si era affatto convinti del successo del dramma. Durante le prove, gli attori scuotevano la testa e si esprimevano assai dubbiosi sulle possibilità sceniche delle tre lunghe sequenze di dialogo nelle quali si sviluppa il dramma. Alla prova generale, cui Strindberg presenziò, si era ancora titubanti [...]. Ma allorché si levò il sipario della prima, arrivò il trionfo. [Rudolf] Rittner, Rosa Bertens e [Josef] Jarno [che nel ruolo di Gustav fu 'scoperto' come attore in quella occasione!] realizzarono una recitazione d'assieme così ben bilanciata, ma nel contempo così ricca di temperamento, che di rado si era e si sarebbe mai vista a Berlino! (Paul I930, 79-83)..5

Ancora una volta, gli attori salvarono un copione anomalo rispetto alle convenzioni sceniche dell'epoca e, anche se la critica tedesca si tenne in generale più riservata del pubblico, già il 26 gennaio del I893 Strindberg poteva scrivere:

Strana amabile nazione questa, che s'inchina al genio straniero, sinceramente, senza resistenza e senza barlume d'invidia. Noi - Drachmann, Heiberg, [il pittore Edvard] Munch, Paul ed io siamo stati di recente a un banchetto da Lehmann, l'editore di [Hermann] Sudermann, il giorno dopo la première [di Heimat] dello stesso Sudermann. Drachmann ha brindato a Sudermann - il grande Sudermann - e costui ha ringraziato, riconoscendo i forti legami con gli scandinavi, i suoi maestri, che stavolta avevano portato das Licht [la luce] (Berlinerwitz Nordlicht) in Germania. Fu un momento memorabile per la storia della letteratura (ASB IX, I23).

“Das Licht kommt jetzt aus Norden!” già, la luce veniva dal Nord: la frase

\footnotetext{
${ }_{13}$ La conferenza è riportata in Paul I930, I90-99.

${ }_{14}$ Insieme agli atti unici del I892 Di fronte alla morte (Inför döden) e Primo avvertimento (Första varningen).

Is Rudolf Rittner aveva già interpretato Jean alla Freie Bühne.
} 
di Sudermann fu ripresa dai giornali e diventò proverbiale ed emblematica di quel momento culturale (cfr. Paul I930, 75), nel quale si diffuse addirittura la moda, fra gli intellettuali tedeschi, di attribuirsi pseudonimi nordici (Gravier I949, 6). Parallelamente, è pur vero però che i nordici, alla fin fine, venivano accolti quasi bruciandosi a vicenda: Ibsen dopo Bjørnson e Strindberg dopo tutt'e due. Infatti, Stefan Zweig - confermando quel che scrive Weininger - ha rievocato che, nella sua gioventù (al trapasso del secolo, quindi), Ibsen già appariva confinato fra "i buoni e solidi maestri" di un'epoca tramontata:

\begin{abstract}
Ogni giorno inventavamo nuovi metodi per utilizzare le ore più noiose della scuola in letture private; mentre il professore esponeva il suo logoro commento intorno alla "poesia ingenua e sentimentale" di Schiller, noi sotto i banchi leggevamo Nietzsche e Strindberg, i cui nomi non erano ancor giunti al vecchio insegnante (Zweig I979, 38). ${ }^{16}$
\end{abstract}

Dopo questo picco di successo, però, anche a Strindberg capitò di registrare quella che avrebbe mestamente definito "una certa stasi" con la constatazione che "il dramma prese di nuovo a ripiegare su posizioni più o meno vecchie” (ASV LXIV, I2-I3). Strindberg in Germania restò soprattutto affidato, a partire dal I894, all'apostolato del fido quanto discusso traduttore Emil Schering, ma, ai primi del Novecento, Alfred Halm a Breslavia e Max Reinhardt a Berlino (quest'ultimo insieme alla coppia d'attori Emanuel Reicher e Gertrud Eysoldt) avrebbero rilanciato sulle scene il drammaturgo svedese con Rausch ovvero Delitto e delitto (Brott och brott).

Reinhardt, in particolare, terrà alte, ai primi del Novecento, le sorti dei drammaturghi nordici, con memorabili allestimenti gravidi d'atmosfera e polemici intenti nei confronti della tradizione naturalistica. Ricordiamo i suoi simbolistici Spettri ibseniani, con le scene di Edvard Munch, nel I906, per l'inaugurazione dei Kammerspiele (vedi anche Fazio I988, 86-87), assieme agli strindberghiani Danza di morte (Dödsdansen) del ıوı2 e Un sogno (Ett drömspel), realizzato per il Dramaten di Stoccolma, il 28 ottobre I92I. ${ }^{\text {I7 }}$

Strindberg, in particolare, si sarebbe sempre più imposto come un maestro delle nuove generazioni e - venerato da Kafka e citato, in quanto emblema antiborghese, da Ernst Toller nel suo primo dramma, La svolta (Die

i6 Sul relativo declino di Ibsen sulle scene tedesche dopo il I895, vedi anche Eller I9I8, II4, che evidenzia tuttavia che ci si trova di fronte più a una crisi della sua centralità che della sua presenza. Infatti, "dove Ibsen era stato uno dei pochi drammaturghi moderni, ora era uno dei molti", mentre era cresciuta una consistente drammaturgia nazionale.

${ }^{17}$ Fondamentale in merito lo studio di Kvam (I974). La lezione di Reinhardt si può dire abbia inciso sulla stessa tradizione interpretativa scandinava (e, in prospettiva, abbia avuto, per molti versi, una portata europea). Il regista svedese Olof Molander fu, in particolare, influenzato da Reinhardt (cfr. Ollén I982, 25I, 37I). 
Wandlung) del I9I9 - come uno dei padri riconosciuti della corrente espressionistica.

Certo il Berliner Tageblatt aveva avuto da ridire sull'infatuazione nazionale per la cultura scandinava (Holm I957, 394); Ibsen, dal canto suo, aveva assunto - s'è accennato - difensivamente assai presto i panni di un tormentato pangermanista (cfr. anche HIS XIII, II3) e Strindberg aveva dovuto occasionalmente registrare che, in fondo, "gli artisti scandinavi erano particolarmente mal visti in certi influenti ambienti" tedeschi (ASV L, 33). Eppure i nordici, in alcune occasioni, avevano effettivamente tenuto il banco in una Germania che viveva uno straordinario momento di affermazione politica. Come mai?

Secondo Ola Hansson, perché "la Germania era grande, ma priva di letteratura” (Strindberg e Hansson I938, 66); un'opinione estrema, non c'è che dire, ma che doveva essere abbastanza diffusa se lo Strindberg del Chiostro (Klostret, I898), romanzo autobiografico in parte ambientato a Berlino, si può permettere di rimetterla sulla bocca di una giornalista austriaca (la sua futura moglie Frida Uhl): “Dopo la guerra del ı870, l'Impero germanico era carente di arte, scienza e letteratura. Lei stessa leggeva solo libri francesi e scandinavi e la giovane letteratura tedesca che stava fiorendo derivava dal Nord" (ASV L, I3).

6. Di contro, la Francia, umiliata proprio dalla potenza bismarckiana, era inevitabilmente più chiusa in se stessa e ciò si può ben rilevare, negli anni Novanta, nell'accanito dibattito (più acre ed esteso di quello tedesco) a proposito dell'influsso scandinavo sulla cultura e il carattere francesi; un dibattito sostanzialmente solcato dal solito sciovinistico pregiudizio verso una corrente che, non a caso, la splendida Sarah Bernhardt bollava, sprezzante, come "Norderie" (cit. in Meyer I97I, 573). La capitale francese, per di più, non era solo una splendida città con molti luoghi di spettacolo, ma un vero e proprio sistema teatrale. Un sistema abbastanza chiuso e autonomo, però. James Brander Matthews scrive nel ı880:

I teatri di Parigi, più o meno una cinquantina se includiamo le sale dei sobborghi, richiedono una fornitura costante di nuovi drammi.

Un drammaturgo francese ha oggi un grande vantaggio sui drammaturghi di ogni altro paese: non conosce concorrenza straniera. Grazie all'abilità degli autori francesi, il loro lavoro è stato esportato in ogni capitale del mondo civilizzato.

[... Ma] potrei ricordare appena due drammi stranieri recitati a Parigi lo scorso anno (I879), un'opera comica tedesca e una tragedia italiana. [...] Nonostante tutto il suo cosmopolitismo, Parigi non dà alcun segnale di disaffezione nei confronti della drammaturgia di casa. [... E] quasi centocinquanta drammi originali 
vengono presentati ogni anno nei venti principali teatri parigini (Brander Matthews I880, 204-07).

Era difficile penetrare in questo mondo autoreferenziale, che, tuttavia, nell'ultimo scorcio del secolo, cominciò a essere percorso da fermenti d'inquietudine, rinnovamento e sperimentazione, che trovarono espressione, nel ı887, nel Théâtre Libre di André Antoine e, nel I893, nel simbolista Théâtre de l'œuvre di Aurélien Lugné-Poe. Non a caso, furono proprio queste scene a guardare a un repertorio internazionale e a spianare la strada agli autori nordici.

Fu indirettamente, attraverso l'apostolato di Edmund Gosse, che i francesi, nel ı873-74, ebbero le prime notizie su Ibsen, grazie agli articoli di Léo Quesnel su La Revue politique et littéraire. Teatralmente, invece (all'apogeo della fortuna tedesca di Ibsen), in Francia, cominciò André Antoine con Les Revenants, il 30 maggio I890, cui seguì Le Canard sauvage, il 28 aprile I89I. $\mathrm{Al}$ di là del coraggio e della curiosità culturale di Antoine, che approccio aveva costui nei confronti di un fenomeno per tanti versi 'alieno' quale poteva considerarsi Ibsen?

Se prendiamo in esame la messinscena di Spettri (che furono, tra l'altro, presentati con tagli che risparmiavano solo il terzo atto), esiste un'attendibile lettera-relazione, inviata a un curiosissimo Strindberg da Ola Hansson, che fu testimone dello spettacolo:

\begin{abstract}
Antoine stesso recitò assolutamente da maestro, con tutto lo spontaneo, illusorio naturalismo gallico e rese il cadavere, nel quale si compiva il processo di putrefazione. Gli altri erano proprio impossibili: Manders un abbé francese dell'ancien régime, la vedova Alving un'eroina tragica, patetica, declamante di un dramma classico francese; Engstrand e Regine recitavano in costumi folcloristici. Il pubblico ridacchiava, dove nel Nord e in Germania avrebbe pianto o avvertito indignazione morale; la stampa era incapace di comprendere come il pubblico (cit. in Ahlström I956, 2I).
\end{abstract}

Del resto, anche un corrispondente del giornale norvegese Morgenbladet, che aveva assistito alle prove, riferisce che la Barny, pur brava, era troppo melodrammatica come Signora Alving, e che gli altri attori erano in maggiore sintonia col testo, senza comunque apparire propriamente adeguati (Nyholm I959, 29-30).

È chiaro che si trattò di uno spettacolo con degli sbilanciamenti piuttosto forti: a prescindere dalla lamentela di Sarcey che gli interpreti non si facevano intendere, ${ }^{18}$ la compagnia non doveva avere né riferimenti né maturità

\footnotetext{
I8 "Antoine, che già non ha voce, parla dando le spalle e in tono basso. Si compiace di
} 
culturale per affrontare l'inedita tessitura dello spartito ibseniano e si barcamenava - con un ardimentoso eclettismo, che si sospetta non infrequente al Théâtre Libre - fra le accettate convenzioni tragiche e le nuove esigenze di documentazione ambientale, quantunque nel finale degli atti primo e terzo riuscisse probabilmente a rendere, con qualche forza patetica, "le scene commoventi a livello mimico" (Pruner I958, 80).

Fra il I893 (a cominciare da Rosmersholm) e il I897, sarebbe stato soprattutto il Théâtre de l'Euvre di Aurélien Lugné-Poe a prodigarsi a favore del drammaturgo norvegese, con una fitta serie di allestimenti, peraltro sostenuti dalla competente, quanto gratuita, collaborazione di Herman Bang, patrocinata dal conte Prozor (Amsinck I972, 7). ${ }^{19}$

In Francia, Ibsen tendeva ora a essere trasfigurato, secondo Maeterlinck (e le celebri versioni francesi del conte Prozor), in un maestro del "dramma da sonnambuli" (Maeterlinck I896, 200). Se dobbiamo credere a Lemaître, già in Le Canard sauvage di Antoine, affiorava la tendenza (forse intuibile anche nei precedenti Spettri), che sarà attribuita ai simbolisti e a Lugné-Poe, di recitare Ibsen "troppo lentamente, troppo seriosamente, troppo maestosamente” (Lemaître I888-98, VI, 3I), a riprova di una percezione insieme inevitabilmente esotica e grave del teatro nordico (che, a ben vedere, male si accordava sia con un testo come L'anitra selvatica sia con la percezione che l'autore aveva del suo stesso stile). ${ }^{20}$

Herman Bang, collaborando con i simbolisti, avrebbe cercato di riportare il norvegese a una cifra nazionale, di "naturalezza e chiarezza", che si sarebbe imposta lentamente e tardi, attorno al I897 (Robichez I957a, 3I-33) ${ }^{21}$. Il regista danese riuscì così ad affermare un determinante interesse, "come principio dell'istruzione scenica, sull'uomo che sta dietro il ruolo", nonché un nuovo conseguente criterio interpretativo, per il quale "l'arte della dizione", tanto coltivata in Francia, "non coincideva con quella dell'attore", sostanziata di ben altri valori umani (cit. in Amsinck I972, I4, I6). Anche questa era "luce che veniva dal Nord" a rinnovare le più fruste convenzioni teatrali, già in autonoma sintonia con certe istanze stanislavskiane.

7. Nel I893, l'anno in cui in Francia si registrò un picco di traduzioni di Ibsen (Shepherd-Barr 20I2, 58), pure August Strindberg e Bjørnstjerne Bjørnson erano arrivati, sulla sua scia, al Théâtre Libre, rispettivamente con Mademoiselle Julie e con Une Faillite, transitando in seguito nel repertorio

risalire il fondo della scena, dove intrattiene a mezza voce lunghe conversazioni con il compagno. Sembrerebbe che fosse per cogliere più da vicino la natura, come se fosse più naturale mostrare le spalle piuttosto che il viso e discorrere a bassa voce invece di farsi sentire" (Sarcey I900-02, VIII, 330-3I).

I9 Amsinck (1972, I3) evidenzia che, a Parigi, nei teatri da boulevard come in recite private, non si disdegnava l'occasionale rappresentazione degli autori nordici.

${ }^{20}$ Lo stesso Aurélien Lugné-Poe (I936, 80-8I) non tace le riserve di Ibsen sullo stile dell'Euvre.

${ }_{21}^{21}$ Nel I895, Bjørn Bjørnson, figlio di Bjørnstjerne, subentrerà a Bang come collaboratore di Lugné-Poe nell'allestimento degli autori nordici. 
del Théâtre de l'EEuvre. Bjørnson, considerato “il rivale di Ibsen”, era stato tradotto in francese con l'antica commedia Les Nouveaux mariés ovvero Gli sposini fin dal I884 (Antoine I921, 293; Reque I930, 26) e, già nel I870, Schuré l'aveva considerato l'esponente più significativo della nuova letteratura nordica. Tuttavia, dopo l'affermazione della drammaturgia borghese di Ibsen, anche in Francia, quando si rappresenterà Une Faillite al Théâtre Libre, "fece la figura di non essere altro che un buon allievo di Augier" (Lescoffier I932, 356). ${ }^{22}$

Per contro, il 2I giugno I894, l'allestimento di Créanciers di Strindberg, curato e adattato con cura sapiente da Herman Bang e con l'interpretazione di Lugné-Poe, Lucienne Dorcy e Philippe Rameau, noti attori dell'Odéon, fu reputato - sebbene preceduto dalla solita poco penetrante conferenza (che Strindberg, tra l'altro, non gradì troppo, visto che gli dava senza tanti complimenti del "barbaro") ${ }^{23}$ - un passaggio cruciale dell'avventura del teatro scandinavo in Francia, tanto da far decretare a Le Figaro: "La Scuola dei Nordici ha avuto la sua serata d'Hernani” (Ahlström I956, 2II, 323). ${ }^{24}$

Soirée d'Hernani o meno, il caso della rappresentazione del I6-I7 gennaio I893 da parte di Antoine della Signorina Julie ci pare emblematico della difficoltà di cogliere storiograficamente la qualità della rappresentazione e il reale impatto delle opere dei nordici sulla scena francese del tempo.

Nell'allestimento di Antoine, la protagonista Eugénie Nau era affiancata da Alexandre Arquillière, che - a detta del suo direttore - fu "straordinario come servo della signorina Julie” (Antoine I921, 288). Nel I92I, Antoine assicurava che il testo era stato allestito "tenendo conto nella maniera più scrupolosa delle indicazioni di Strindberg”, sicché la scenografia della cucina, nella quale è ambientata l'azione, "secondo il suo desiderio, 'si perdeva nelle quinte', in modo, secondo l'autore, che ci sia posto per le suggestioni e che l'immaginazione stimolata dallo spettacolo la completi da sé" (Antoine I932, 232); appese c'erano pure 46 casseruole e a destra si notava una porta a vetri che lasciava intravedere un giardino; soppresse erano le bande laterali che incorniciavano la scena. La documentazione sulle tecniche di allestimento nei primi anni del Théâtre Libre è piuttosto carente, ma, nel caso della Signorina Julie, resta un disegno di Ernest Clair-Guyot, su Le Monde artiste del 29 gennaio I893, che ci conferma l'accuratezza formale dell'allestimento e ci mostra la protagonista, nella conclusione, con in mano il raso-

22 Quantunque parzialmente si rifacesse, "nonostante un'interpretazione imperfetta nel suo fervore”, l'anno successivo, all'Euvre con Au-dessus des forces humaines ovvero Al di là delle nostre forze (Over Evne, I883) (Lescoffier I932, 356).

${ }_{23}$ In un articolo su Gil Blas dell'8 agosto I895, Il barbaro a Parigi (Le Barbare à Paris), Strindberg avrebbe reagito alla presentazione del letterato Henri Chantavoine, che riporta in questi termini: "Niente di speciale ciò che state per vedere, niente proprio: un orso polare, una foca, non saprei come dire: proprio un barbaro" (ASV XXXIV, 260).

${ }^{24}$ Il I3 dicembre I894, il Théâtre de l'Euvre avrebbe presentato con un certo successo anche Le Père di Strindberg, con un finale un po' attenuato. 
io, irrigidita, di fronte al servo, che imita un po' platealmente i movimenti di un ipnotizzatore (cfr. Ahlström I956, I77).

Mademoiselle Julie era stata presentata con À bas le progrès di Edmond de Goncourt e Le Ménage Brésil di Coolus, provocando, secondo Antoine,

in fondo una sensazione enorme. Tutto ha appassionato il pubblico, il tema, l'ambientazione, l'atto unico di un'ora e mezzo che stringe un'azione bastante ad alimentare una grande pièce francese. Certo ci sono state delle sghignazzate, delle proteste, ma, alla fine, ci si è trovati di fronte a ben altro (Antoine I92I, 286-88).

A differenza del testo di Coolus, quello di Goncourt fu accolto con indifferenza (pare che alla spicciolata il pubblico abbandonasse la sala) e si suppone che l'autore si fosse danneggiato da solo, facendo pubblicare, il i6 gennaio, su L'Écho de Paris e Le Rappel, una bellicosa dichiarazione contro Tolstoj e l'“idolatria dei giovani drammaturghi per il teatro scandinavo" in nome delle "qualità francesi: la chiarezza, lo spirito, l'ironia"; un attacco che - osserva tristemente Antoine (I921, 286-88) - lo faceva passare dalla parte dei suoi peggiori avversari, Sarcey e Pessard. L'intervento sollevò una polemica giornalistica, ma appariva in effetti tanto inopportuno quanto più Strindberg, nella prefazione distribuita al pubblico, aveva cavallerescamente indicato i Goncourt fra gli ispiratori del proprio lavoro (cfr. ASV XXVII, IO9).

Con Birger Mörner, 1'8 febbraio I893, Strindberg avrebbe commentato così la sua avventura parigina:

Non è vero che La signorina Julie è caduta (che fosse abbattuta da quel 'vecchio somaro' di Sarcey e simili era ovvio); vero è invece che Goncourt ha fatto un tonfo con il suo stupido dramma $\grave{A}$ bas le progrès (indirizzato contro Ibsen e me). Così io ho battuto il primato di Goncourt e questo ha irritato i suoi amici - hinc (ASB IX, I28).

Da un'altra lettera di poco successiva allo stesso Mörner, risulta che anche Antoine si sarebbe "complimentato" con il drammaturgo "per il successo" (ASB IX, I34).

A un'analisi più attenta, però, è difficile sostenere che La signorina Julie fosse stata tanto bene accolta. Ciò che racconta Antoine, infatti, appare piuttosto roseo e Ginisty nota per contro: “Non è stata una gran serata per il Théâtre Libre” (cit. in Ahlström I956, I68). Il conservatore Sarcey ribadì di non capire Strindberg e lo ribattezzò, ironico: "génie norvégien”. Se questo non stupisce, le altre critiche furono in linea di massima piuttosto aspre: "Strindberg è riuscito a creare a Parigi lo stesso fenomeno che crea in patria: 
conflitto" (Ahlström I956, 312), fu il commento di un giornalista svedese. Su La République Française del 23 gennaio I893, Ginisty puntualizza:

Il disastro è stato irrimediabile, nonostante il valore degli interpreti Nau e Arquillière, che hanno fronteggiato arditamente la tempesta. Immagino che Strindberg ora sia particolarmente interdetto. Perché si accetta Ibsen e non lui? Si riscontra subito una differenza essenziale tra i due scrittori: l'opera ibseniana è pregna di poesia. Di contro, Strindberg sembra voler proscrivere la poesia dal suo teatro, respingendola come una seduzione pericolosa, indegna d'un uomo che ha le mani piene di verità amare (Ahlström I956, I68, 313).

Il concetto di 'successo' per gli autori nordici e i laboratori d'avanguardia che li rappresentavano a Parigi, come si vede, poteva essere alquanto opinabile.

Nonostante un'obiettiva crisi di allestimenti a partire dal I897 (la cosiddetta fortuna europea dei nordici si conferma sempre piuttosto alternata) e, nonostante lo spiritoso epitaffio di Maurice Muret ai primi del secolo ("Il polo è passato di moda"; Reque I930, 203), almeno in certe correnti apparentemente laterali della scena francese, il teatro scandinavo ha mantenuto, nel Novecento inoltrato, un suo ruolo seminale. Soprattutto Strindberg, diremmo. Basti pensare al comunque eccezionale Songe allestito nel ig28 da Antonin Artaud, ma, oltre, al suo influsso sulla corrente del Teatro dell'Assurdo e segnatamente su Samuel Beckett. È emblematico, infatti, che, nonostante il riserbo che Beckett ha potuto dimostrare difensivamente nei confronti della scrittura del drammaturgo svedese, ${ }^{25}$ Roger Blin (allievo di Artaud) abbia sottolineato che fu "grazie a Strindberg che era venuto in contatto con Samuel Beckett”, allorché, al Théâtre de la Gaîté-Montparnasse, nel i949-50, aveva tentato un allestimento di Sonata di spettri (Spök-Sonaten):

Lo spettacolo non andava bene. C'erano pochi spettatori, ma, fra questi, una sera fece la sua comparsa Beckett, che per di più tornò una seconda volta. Dopo qualche tempo, ricevetti una visita di sua moglie Suzanne, che mi portò il manoscritto di En attendant Godot. Beckett aveva evidentemente apprezzato la mia interpretazione strindberghiana e ne aveva dedotto che fossi in grado di comprendere anche lui e di portarlo in scena (Blin I967, II). ${ }^{26}$

8. "C’è qualcosa di Shakespeare in questo anarchico" (Reque i930, 58), aveva scritto a proposito di Ibsen, il 30 maggio I89o, su Le Figaro, Paul Des-

25 Nel i967, Beckett ha dichiarato: "Non conosco bene il teatro di Strindberg e non posso esserne stato influenzato" (cfr. Swerling I971, 26).

${ }_{26}$ Ricordiamo incidentalmente che, nel I955, persino Arthur Adamov avrebbe pubblicato una discontinua monografia su Strindberg (Adamov I955). 
jardins, dopo Les Revenants dati da Antoine, e la rassegna di spettacoli degli ultimi decenni dell'Ottocento, che abbiamo delineato, dall'esito qualche volta luminoso e spesso contrastato, che si sono imposti comunque progressivamente alla memoria storica come un'affermazione di valori scenici nella coscienza teatrale europea, non dissuade dal fissare l'idea che l'epoca di Bjørnson, Ibsen e Strindberg costituì una specie di 'età elisabettiana' 'oscariana' dovremmo definirla! - o di siglo de oro del teatro nordeuropeo che doveva inevitabilmente avere riflessi continentali.

Si trattò di un trentennio (dal I870 circa) bene individuabile e fecondo, che, a fianco dei suoi Shakespeare e Marlowe, contò pure i più piccoli Greene o Beaumont e Fletcher, che ovviamente avevano più difficoltà a figurare sia in Germania sia nella luccicante vetrina parigina, ${ }^{27}$ nonché i suoi grandi interpreti e allestitori (ingiustamente in ombra nella manualistica teatrale corrente). Questo momento segnò certo per la Scandinavia uno spartiacque: il suo ingresso nella cultura e nella coscienza europea moderna e il prospettico consolidarsi di uno stile che, a suo modo, con Lars von Trier o Jon Fosse, appare ancora oggi ben distinto.

La circostanza singolare è che tanta fioritura non aveva radici in un humus culturale o teatrale peculiarmente rigoglioso. Anzi. Nel Settecento, Ludvig Holberg aveva ammesso: "Noi nordici siamo i fedeli imitatori dei francesi" (Holberg I969-7I, XI, 247), e, dopo tutto, per trovare un'altra opera svedese rappresentata a Parigi prima di Strindberg, si deve risalire al I803 ovvero a una commedia di re Gustavo III, Siri Brahe ou les curieuses, alla Comédie Française. Così, gli autori scandinavi di fine secolo dovettero fronteggiare e, ironicamente, condividere l'opinione corrente di essere dei 'Barbari della cultura', nell'accezione distruttiva, ma anche rigeneratrice, che il termine storicamente implica.

Nel I893, Darzens, un intellettuale favorevole a Strindberg, rilevava che "i critici si scagliano contro questa invasione 'barbarica' che minaccia di scompaginare le sane tradizioni francesi”, che costituivano la norma teatrale occidentale (Reque I930, I48-49). Due anni più tardi, lo stesso Strindberg avrebbe pubblicato il menzionato articolo Le Barbare à Paris (vedi nota 23), sottolineando - da "Normand" pur accolto nella cerchia dell'Euvre di LugnéPoe - non solo la personale non facile integrazione, ma anche certi secolari rapporti culturali e politici che legavano il suo paese a "la Mecque d'Occident”. L’Europa, pare insinuare saggiamente Strindberg, nasce comunque

${ }_{27}$ Per esempio, nel I894, l'eminente critico e drammaturgo danese Edvard Brandes passò quasi inosservato con Una visita (Et besøg) sulla scena sperimentale de Les Escholiers. LugnéPoe annunciò invece messinscene di Tor Hedberg e Knut Hamsun, ma, se si eccettuano Delinquente (Förbrytare) di Gustaf af Geijerstam, dato solo in tournée a Stoccolma nel I894, e l'adattamento di un'oscura novella di Ellin Ameen firmato dal conte Prozor due anni dopo, giusto Herman Bang, con Fratelli (Brødre), e il notevole drammaturgo norvegese Gunnar Heiberg, con Il balcone (Balkonen), furono presenti, nel I894 e '98, sui cartelloni dell'Euvre (si veda in merito Robichez I957b). Nessuno di questi testi peraltro sollevò echi memorabili. 
dalla fusione dello spirito latino con la 'barbarie' nordica: "Comincio a sentirmi un po' a casa, fiero di sapere che i miei antenati hanno contribuito alla gloria scientifica e artistica della Ville-Lumière - che oggi mi tratta come un intruso, un pellerossa, da antropomorfo" (ASV XXXIV, 263).

Si può affermare che i grandi drammaturghi scandinavi - proprio dalla frizione e dal contrasto con la cultura continentale - avvertirono più acutamente e orgogliosamente l'eccezionalità della loro avventura scenica, che li portava a imporsi non solo su differenti e assai aliene sensibilità, ma addirittura in nazioni che avevano nutrito e, per certi versi, 'colonizzato' la non ricca storia teatrale che avevano alle spalle. In parallelo, gli autori nordici davano a un'Europa intellettuale, segmentata in arcigni nazionalismi quanto quella politica, una coscienza più aperta e più oggettiva della sua intima costituzione culturale e, anche per questo, l'affermazione di Ibsen o di Strindberg non poteva essere una marcia trionfale.

I drammaturghi scandinavi accettavano di norma, in Francia, di essere rappresentati senza alcun compenso, pur di comparire sui cartelloni di quei 'laboratori' poveri, ma prestigiosi per il loro riconosciuto ruolo d'avanguardia, che erano i teatri di Antoine e di Lugné-Poe (Amsinck 1972, 42-43). Va da sé poi che il successo garantito da queste scene restava d'élite e scalfiva il sistema degli spettacoli solo obliquamente: la vita teatrale parigina - quella commerciale che si articolava prospera nei tanti luccicanti teatri della capitale - era ben lungi dall'inchinarsi incondizionatamente ai nordici. Infatti, la Comédie Française arriverà a rappresentare Ibsen solo nel I92I e - si è di recente giustamente notato - che al limite un'affermazione davvero 'popolare' del norvegese può considerarsi Maison de poupée con Réjane, nell'aprile del I894, al Théâtre du Vaudeville, al di fuori cioè dei teatri sperimentali (Shepherd-Barr 20I2, 62-63).

Questo solo potrebbe aprire un ampio discorso su che cosa s'intenda per 'affermazione' o per 'conquista' di una stabile posizione nei repertori internazionali riferita ai massimi rappresentanti della drammaturgia nordica. In questa sede, ci limiteremo a osservare che verosimilmente si trattò - sia in Germania sia in Francia - di un'acquisizione insieme cronologicamente concentrata eppure discontinua, rappresentabile in un grafico con picchi e lunghe depressioni. Peraltro, la ricezione di Ibsen, Bjørnson e Strindberg da parte degli intellettuali e degli uomini di teatro più avanzati, nonché da parte della storiografia stessa, non corrisponde (ancor oggi) al loro effettivo impatto sul pubblico (al di là, ovviamente, delle contingenti realtà locali norvegese e svedese, che non appaiono neppure 'costantemente' tanto ricettive, se analizziamo l'arco dell'ultimo secolo e mezzo).

Insomma, per intenderci, avranno avuto senz'altro ragione sulla lunga prospettiva i sostenitori del coraggioso Antoine che, nel I889, prevedevano che "l'édifice vermoulu" (bell'aggettivo ibseniano) del teatro commerciale e tradizionale, sotto i colpi del Théâtre Libre, sarebbe crollato (così, nel I889, 
Maurice Lefèvre, cit. in Antoine I979, I72), ma se vogliamo considerare la storia nel complesso ovvero nella sua infinita e paradossale complessità almeno dal punto di vista del 'successo di pubblico' - non bisognerebbe mai dimenticare che non furono né Spettri né, peggio, La signorina Julie i testi più rappresentati e seguiti nell'Ottocento, bensì la riduzione di Le Tour $d u$ monde en quatre-vingts jours di Jules Verne, che fra il I874 e il I898 totalizzò ben i500 repliche (Weber I990, I98-99).

Pertanto, sussiste inevitabilmente uno iato fra l'acquisizione di un fenomeno artistico particolarmente raffinato nell'ambito culturale e il successo diffuso e, in questo intervento, abbiamo avuto modo di trattare più dell'acquisizione che del successo, ma anche in fondo del sostanziale contributo dei ‘barbari’ nordici all'identità europea.

\section{BIBLIOGRAFIA}

Adamov, Arthur. I955. August Strindberg, dramaturge. Paris: L’Arche.

Ahlström, Stellan. I956. Strindbergs erövring av Paris. Stockholm: Almqvist \& Wiksell.

Amsinck, Hanne. 1972. Sceneinstruktøren Herman Bang og det franske symbolistiske teater. København: Gad.

Antoine, André. I92I. Mes souvenirs sur le Théâtre Libre. Paris: Arthème Fayard.

—. I932, Le Théâtre II. Paris: Les Éditions de France.

—. I979. Le Théâtre Libre. Paris / Genève: Slatkine.

Blin, Roger. I967. "Vitnesbyrd." Teatrets teori og teknikk 5: II-I4.

Brander Matthews, James. I880. The Theatres of Paris. London: Sampson Low, Marston, Searle \& Rivington.

Brandes, Georg. I977. Henrik Ibsen. Bjørnstjerne Bjørnson. New York: Arno Press.

Eller, William Henri. I918. Ibsen in Germany, 1870-1900. Boston: Richard G. Badger. Fazio, Mara. I988. Lo specchio, il gioco e l'estasi. Roma: Levi.

Gravier, Maurice. I949. Strindberg et le théâtre moderne. L'Allemagne. Lyon / Paris: IAC.

Grube, Max. I963. The Story of the Meininger, a cura di Wendell Cole. Coral Gables: University of Miami Press.

Holberg, Ludvig. I969-7I. Vorker, voll. I-XII, a cura di Frederik Julius Billeskov Jansen. København: Rosenkilde \& Bagger.

Holm, Ingvar. I957. Ola Hansson. En studie i åttitalsromantik. Lund: Gleerup.

Ibsen, Henrik. I898-I902. Samlede Vorker, voll. I-X, a cura di Jens Braage Halvorsen e Halvdan Koht. København: Gyldendal.

—. I909. Efterladte skrifter, voll. I-III, a cura di Halvdan Koht e Julius Elias. Kristiania / København: Gyldendal.

—. 2005-IO. Skrifter, voll. I-XVII, a cura di Vigdis Ystad et al. Oslo: Aschehoug. 
Koht, Halvdan. I954. Henrik Ibsen. voll. I-II. Oslo: Aschehoug.

Koller, Ann Marie. I965. "Ibsen and the Meininger." Educational Theatre Journal I7: IOI-IO.

-. I984. The Theater Duke. Georg II of Saxe-Meiningen and the German Stage. Stanford: Stanford University Press.

Kvam, Kela. I974, Max Reinhardt og Strindbergs visionoere dramatik. København: Akademisk Forlag.

Lemaître, Jules. I888-98. Impressions de théâtre. voll. I-X. Paris: Lecène.

Lescoffier, Jean. I932. "Pour lire Björnson." Mercure de France, I dicembre: 355-68.

Lugné-Poe, Aurélien. I936. Ibsen. Paris: Rieder.

Maeterlinck, Maurice. I896. Le Trésor des humbles. Paris: Mercure de France.

Melchinger, Siegfried. I962. "German Theatre People Face to Face with Strindberg." World Theatre. Le Théâtre dans le monde II: 3I-44.

Meyer, Michael. I97I. Ibsen. En biografi. Oslo: Gyldendal.

Nyholm, Kela. I959. "Henrik Ibsen paa den franske scene.” Ibsen-Årbok I957-59: 7-78.

Ollén, Gunnar. I982. Strindbergs dramatik. Kristianstad: Sveriges Radios Förlag.

Osborne, John. I988. The Meiningen Court Theatre, 1866-1890. Cambridge: Cambridge University Press.

Paul, Adolf. 1930. Min Strindbergsbok. Stockholm: Norstedt.

Pruner, Francis. 1958. Le Théâtre Libre d’Antoine. Le Répertoire étranger. Paris: Lettres Modernes.

Reque, A. Dikka. I930. Trois auteurs dramatiques scandinaves, Ibsen, Björnson, Strindberg, devant la critique française 1889-1901. Paris: Champion.

Robichez, Jacques. I957a. "L'Introduction de l'œuvre d'Ibsen en France, I887-I889." Revue d'histoire du théâtre 9: 23-35.

—. I957b. Le Symbolisme au théâtre. Paris: L'Arche.

Sarcey, Francisque. I900-02. Quarante ans de théâtre (feuilletons dramatiques). voll. I-VIII. Paris: Bibliothèque des annales politiques et littéraires.

Shepherd-Barr, Kirsten E. 20I2. "Ibsen in France from Breakthrough to Renewal." Ibsen Studies 12: 56-80.

Strindberg, August. I947-200I. Brev, voll. I-XXII, a cura di Torsten Eklund e Björn Meidal. Stockholm: Bonnier.

—. I98I-20I3. Samlade verk, voll. I-LXXII, a cura di Lars Dahlbäck et al. Stockholm: Almqvist \& Wiksell / Norstedt.

—, e Ola Hansson. 1938. August Strindberg och Ola Hanssons brevväxling 1888-1892, Stockholm: Bonnier.

Swerling, Anthony, I97I. In Quest of Strindberg. Cambridge: Trinity Lane Press.

Weber, Eugen. I990. La Francia 'fin de siècle', trad. Erica Joy Mannucci. Bologna: Il Mulino.

Weininger, Otto. I985. Delle cose ultime, trad. Fabrizio Cicoria. Pordenone: Studio Tesi.

Zweig, Stefan. I979. Il mondo di ieri, trad. Lavinia Mazzucchetti. Milano: Mondadori. 